Noi se reduct i on ef fect in super-hi gh resol ut i on LCDs usi ng i ndependent sub- pi xel dri vi ng technol ogy

\begin{tabular}{|c|c|}
\hline 著者 & $\begin{array}{l}\text { I chi kawa Kat suhi ro, N shi Yoshi kazu, Hayashi } \\
\text { Shi geo, Hasegawa M ki o, Koder a Yoshi e }\end{array}$ \\
\hline $\begin{array}{l}\text { j our nal or } \\
\text { publ i cat i on title }\end{array}$ & $\begin{array}{l}\text { Pr ogr ess i n Bi onedi cal Opt i cs and I nagi ng - } \\
\text { Pr oceedi ngs of SPI E }\end{array}$ \\
\hline vol une & 6917 \\
\hline year & 2008-01- 01 \\
\hline URL & ht t p: //hdl . handl e. net /2297/10941 \\
\hline
\end{tabular}




\title{
Noise Reduction Effect in Super-high Resolution LCDs using Independent Sub-pixel Driving Technology
}

\author{
Katsuhiro Ichikawa* ${ }^{\mathrm{a}}$, Yoshikazu Nishi ${ }^{\mathrm{b}}$, Shigeo Hayashi ${ }^{\mathrm{b}}$, Mikio Hasegawa $^{\mathrm{b}}$ \\ Yoshie Kodera $^{c}$ \\ ${ }^{a}$ Graduate School of Medical Science, Kanazawa University, 5-11-80 \\ Kodatsuno, Kanazawa, Ishikawa, Japan 920-0942 \\ ${ }^{\mathrm{b}}$ Totoku Electric Co., Ltd., Japan \\ ${ }^{\mathrm{c}}$ School of Health Sciences, Nagoya University, Japan
}

\begin{abstract}
We have developed and reported a super-high resolution liquid crystal display (SHR-LCD) using a new resolution enhancement technology of the independent sub-pixel driving (ISD) that utilizes three sub-pixels in each pixel element. This technology realizes the three-times resolution enhancement of monochrome LCDs, and improves the depiction ability of detailed shape such as micro-calcifications of a mammography and bone structures. Furthermore, the ISD technology brings not only resolution enhancement but also noise reduction effect by the high-resolution data sampling in displaying the clinical images.

In this study, we examined the efficacy of the newly developed LCDs from the noise power spectrum measurement (NPS), the perceptual comparison of the phantom images and the clinical images. A 15 mega-pixel (MP)SHR-LCD out of a 5MP LCD and a 6MP SHR-LCD out of a 2MP LCD were used for the measurement and the evaluation. In the NPS measurements, the noise of all the SHR-LCDs was improved obviously. The improvement degree of the NPS varied according to the sub-sampling ratio of the data sampling implemented during the image displaying, and the 6MP LCD showed higher improvement. In the perceptual evaluation of the quality-control phantom images and the low-contrast images of the micro-calcifications of the mammography, all the SHR-LCDs provided higher performance than the conventional LCDs. These results proved that the SHRLCDs using the ISD technology had the excellent ability to display the high-resolution clinical images.
\end{abstract}

Keywords: liquid crystal display (LCD), noise power spectrum (NPS), independent sub-pixel driving (ISD)

\section{INTRODUCTION}

Recently, the resolution (matrix size) of flat-panel detectors (FPDs) and the computed radiography systems (CRs) have already been beyond approximately 12 mega-pixels (MP). Furthermore, in the advanced digital mammography systems, the resolution reached range from 16MP to 65MP. In these situations, the liquid crystal displays (LCDs) are playing important roles as the main displaying modality in the soft-copy image diagnosis environment. However, even 5MP monochrome LCDs as the highest resolution displays do not have the enough resolution (matrix size) that are equivalent with those of the FPD and CR systems as required. Therefore, radiologists are forced to observe the sub-sampled images on the LCDs, or to operate magnifying function of the image viewing software. In order to improve these situations, we have developed the super-high resolution liquid crystal displays (SHR-LCDs) using a new resolution enhancement technology by means of the independent subpixel driving (ISD)[1]. Each pixel of monochrome LCDs consist of three sub-pixels equivalent to RGB sub-pixels of color LCDs. Driven each sub-pixel by pixel value corresponding to information recorded in the FPD or CR image, the three times resolution enhancement is achieved. Fig.1 shows the resolution enhancement mechanism of the ISD compared with the conventional LCD. From this restrictive technique, the resolution enhancement is realized only in the sub-pixel chain direction. But as shown in Fig.2, the displayed image exhibits the higher

*E-mail: ichikawa@mhs.mp.kanazawa-u.ac.jp, 


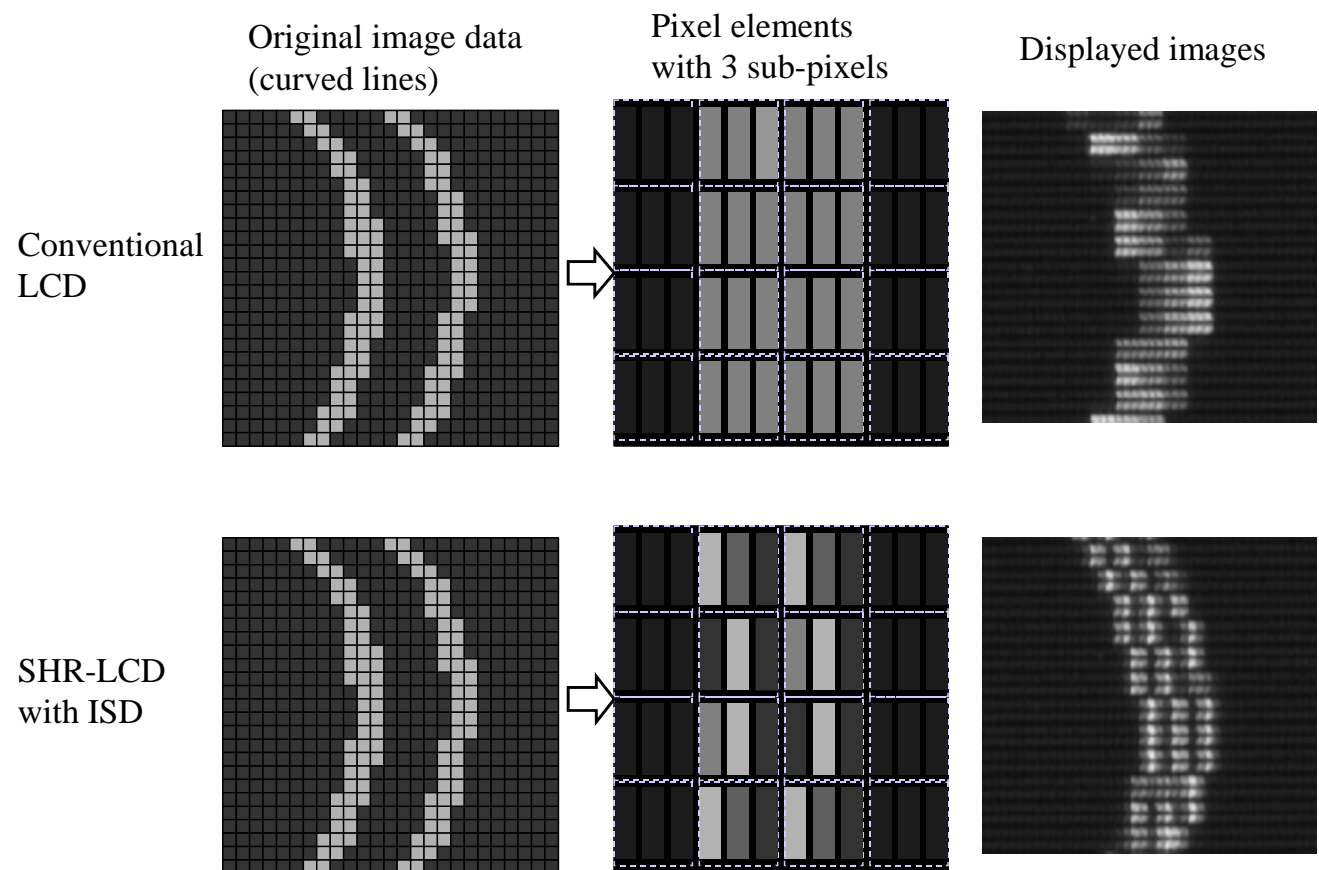

Fig.1. The resolution enhancement mechanism of the SHR-LCD with the ISD technology

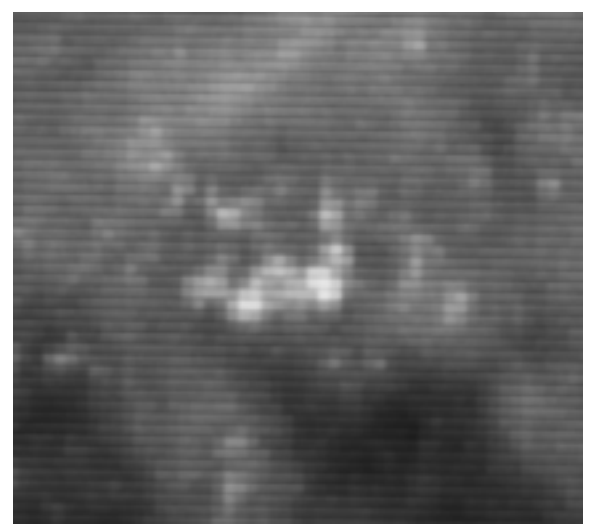

(a)

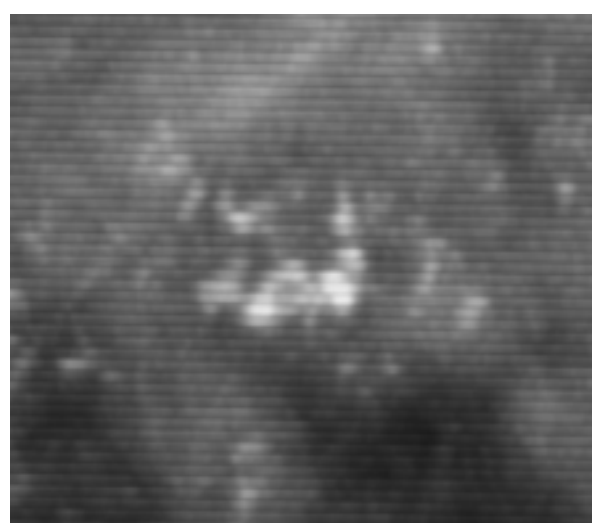

(b)

Fig.2. Displayed clinical image on (a) a conventional 5MP LCD and (b) a 15MP SHR-LCD

Table 1. Matrix sizes of conventional LCDs and SHR LCDs with ISD in case of landscape orientation

Conventional LCD $\rightarrow$ SHR LCD with ISD

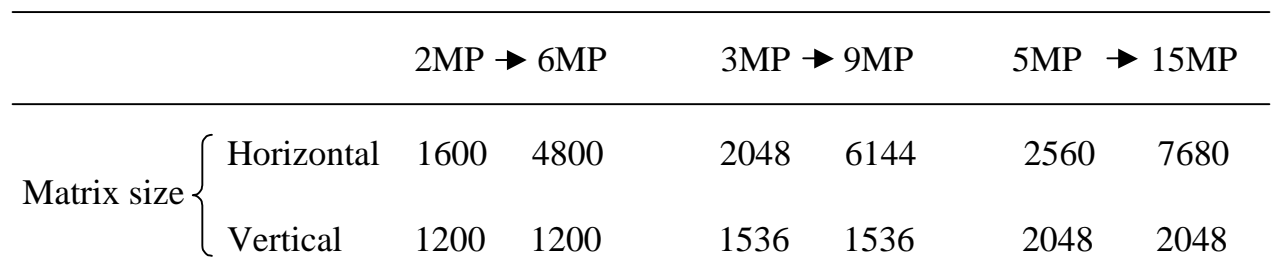


resolution and the shape reproducibility, and does not present any distortions and artifacts by its asymmetric resolution. Thus the new technology realized 6MP SHR-LCD out of a 2MP LCD, 9MP SHR-LCD out of a 3MP LCD and 15MP SHR-LCD out of a 5MP LCD. Table 1 shows the matrix sizes of these LCDs in case of their landscape orientation. When the clinical X-ray images taken by the FPD or CR system, which contain a measure of recognizable noise, are displayed on these SHR-LCDs, we can see not only the resolution enhancement but also the noise reduction effect compared with the conventional LCDs. This noise reduction effect emphasizes the image quality improvement of the SHR-LCD. In order to display a high-resolution image on the SHR-LCD, a tripled high-resolution data-sampling for sub-pixel rendering is implemented in a viewing software. We expected that the noise reduction effect is brought by this high-resolution data sampling, by which the noise aliasing is reduced.

In this study, we quantitatively evaluate the noise reduction effect of the SHR-LCD by means of the noise power spectrum (NPS) measurement using the noise image obtained from digital X-ray systems, and then confirmed this effect through perceptual comparisons by the clinical images and the phantom images.

\section{METHODS AND MATERIALS}

\subsection{LCDs and measured images}

A 15 mega-pixel (MP) SHR-LCD and a 6MP SHR-LCD were measured and examined. Both the LCDs were monochrome type and were provided with a function of switching between the ISD mode and the normal mode. With the normal mode, the resolutions of respective LCDs are 5MP and 2MP. For obtaining noise images for the NPS measurement, an ACR (the American College of Radiology)-156 phantom for mammographic image evaluation was imaged by using two CR mammography systems: Profect CS (Fuji medical systems, Japan) and Mermaid (Konica Minolta, Japan), which have the pixel size of $0.05 \mathrm{~mm}$ and $0.025 \mathrm{~mm}$ respectively.

The radiation dose defined as the mean glandular dose for both CR systems was set to $1.5 \mathrm{mGy}$ equivalent to midrange dose in clinical images. Then, those images were displayed on respective LCDs by the ISD mode and the normal mode in specified magnifying conditions. The window widths for both CR systems were adjusted so that enough noise amounts could be obtained, and the window levels are adjusted so that the luminance became to be arpproximately $100 \mathrm{~cd} / \mathrm{m}^{2}$ in the center of the target area.

\subsection{Measurements of native NPS for LCDs}

We have performed the measurements of the native NPS for the used LCDs, not displaying the noise image. A uniform image with 50\% digital drive level (DDL) was displayed, and captured by a single-lens reflex type digital camera (Nikon D80) in the maximum magnification condition in order to eliminate the aliasing errors caused by insufficient sampling pitch. Then the NPSs were calculated from its CCD data [2]. For the NPS calculation, we employed a one-dimensional method, in which a 1-D noise profile is extracted from a target-data by scanning with a numerical synthesized slit with height of about 30 to 50 pixels [3-6]. The 1-D noise profile was processed by a Hanning window in order to reduce the frequency leakage in the Fourier analysis.

\subsection{NPS measurements using noise images}

We utilized the uniform areas between the test objects such as simulated fibrils and the masses in the images of the ACR-156 phantom. The areas were captured by the digital camera by the same conditions used for the measurement of the native NPS. Then the NPSs were calculated as well. In order to reduce the statistical errors, each NPS result was calculated by averaging the results of three images.

\subsection{Sub-sampling ratio in NPS measurement}

It is supposed that the noise reduction effects on SHR-LCDs are brought by the reduction of the noise aliasing. In fact, the effects appear when the image is displayed in small sub-sampling ratio conditions. When the images of the Profect CS and Mermaid were displayed by fitting to display-area of LCD screen with the two-image layout, the sub-sampling ratios for 5MP LCD were 0.35 and 0.18 respectively, and the ratios for the $2 \mathrm{MP}$ were 0.21 and 0.11. Therefore we measured the NPSs using these small sub-sampling ratios. Also the variance of the noise reduction by changing the sub-sampling ratio was examined. 


\subsection{Perceptual evaluation}

In the perceptual evaluations, the clinical mammography images and the images of the ACR-156 phantom were displayed on respective LCDs. The sub-sampling ratios were set to the above-mentioned fit conditions. Then we perceptually compared the image displayed in the normal mode and the ISD mode.

\subsection{Native NPSs of the LCDs}

\section{Results}

Fig. 3 shows the results of the native NPS for respective LCDs. The resultant NPSs of both the normal mode and the ISD mode were in good agreement.

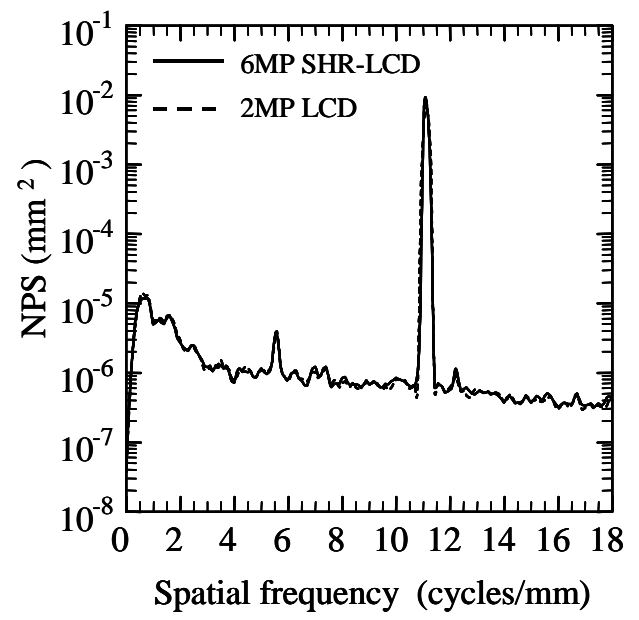

(a)

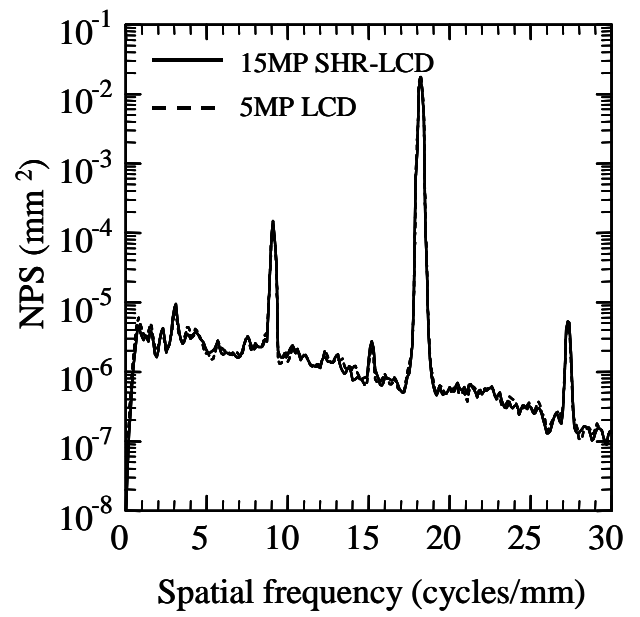

(b)

Fig.3. Resultant native NPS of LCDs. The NPSs of the normal mode and the ISD mode were in good agreements because the drive levels of both modes are perfectly same by the LCD's drive mechanism.

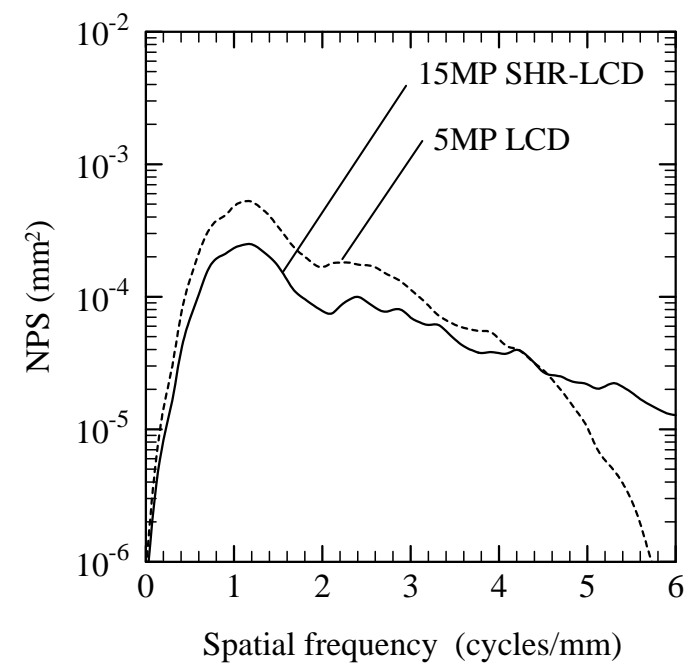

(a)

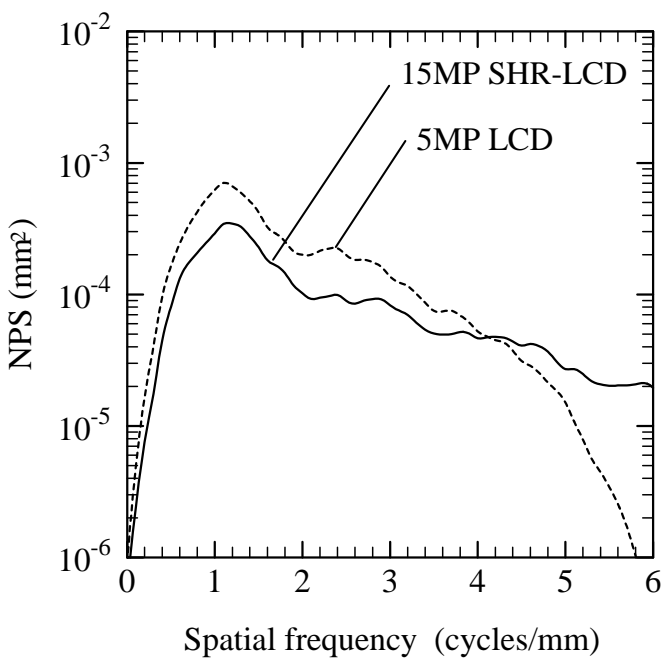

(b)

Fig.4. Comparisons of NPSs measured from the displayed noise images of (a) Profect CS and (b) Mermaid.

The sub-sampling ratios were set to 0.35 and 0.18 respectively on the 5MP LCD. The ratios for $15 \mathrm{MP}$ LCD were determined so that the displayed image sizes of both were the same. 


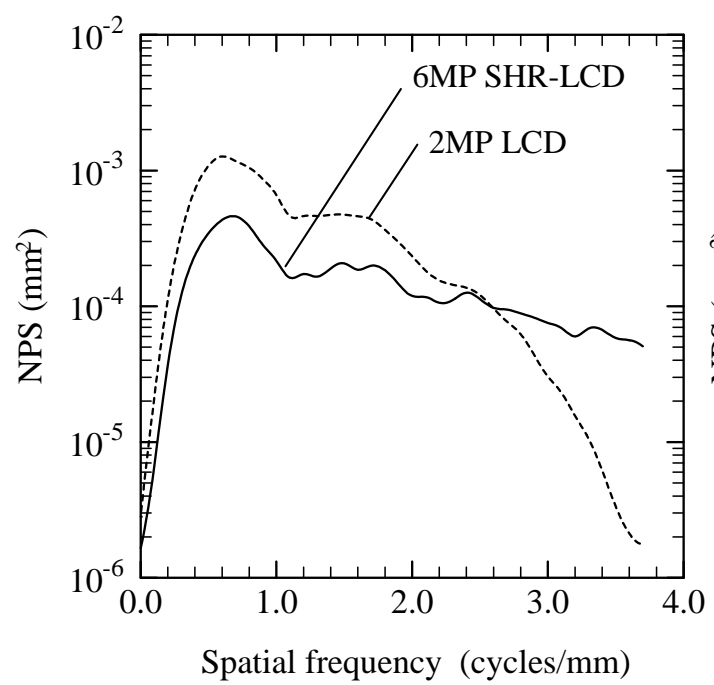

(a)

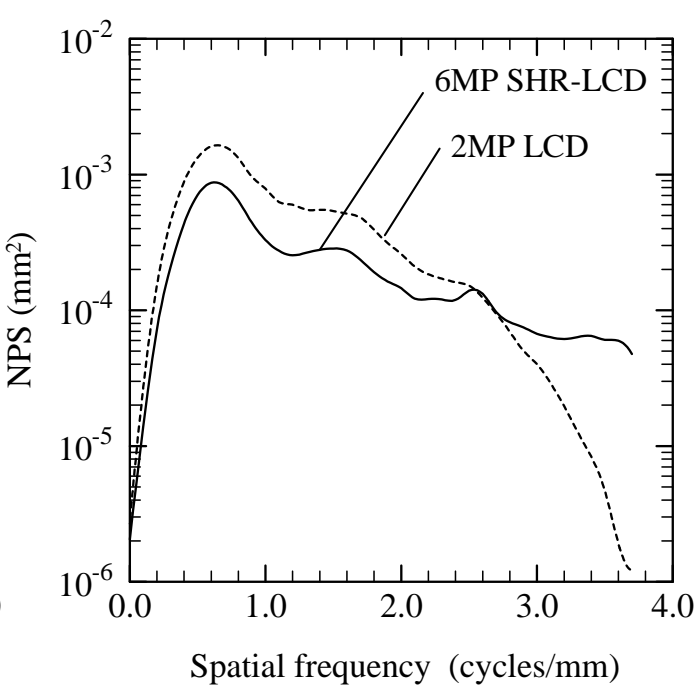

(b)

Fig.5. Comparisons of NPSs measured from the displayed noise images of (a) Profect CS and (b) Mermaid. The sub-sampling ratios were set to 0.21 and 0.11 respectively on the 2MP LCD. The ratios for 6MP LCD were determined so that the displayed image sizes of both LCDs were the same.

\subsection{Results of NPS measurements using the noise image}

Fig. 4 and Fig. 5 show the comparisons of the NPSs between the normal mode and the ISD mode in the specified sub-sampling conditions. All the results represent the NPSs in the horizontal (sub-pixel chain) direction. The spatial frequency scales were calculated by using the actual sizes on the LCDs. In all comparison results, the ISD showed significant noise reduction, and the improvement degrees of the 6MP SHR-LCD were better than those of the 15MP SHR-LCD. In these small sub-sampling conditions, the reductions were recognized in the low frequency below 4.2 cycles $/ \mathrm{mm}$ for $15 \mathrm{MP}$ and 2.5 cycles $/ \mathrm{mm}$ for $6 \mathrm{MP}$. But, in the frequency above that frequency, the relations between two modes were inverted. Fig. 6 shows the change of reduction degree by the sub-sampling ratio for 15MP SHR-LCD. The improvement of the NPS was mostly disappeared at a sub-sampling ratio of 0.55 .

\subsection{Perceptual evaluation of the displayed clinical images and the phantom images}

Fig. 6 and Fig.7 show the captured micro-calcification images of a mammography displayed on the screens of respective LCDs. The used clinical mammography images were obtained by Profect CS. The sub-sampling ratio was set to 0.35 for $5 \mathrm{MP}$. The ratio on 15MP was determined so that the displayed image sizes of both 5MP and $15 \mathrm{MP}$ were the same. For $2 \mathrm{MP}$, the ratio was set to 0.21 , and the one for $6 \mathrm{MP}$ was determined as well. Both the 15MP SHR-LCD and the 6MP SHR-LCD remarkably suppressed the image noise corresponding to the results NPSs. Fig.8 shows the captured images of the simulated micro-calcifications and the masses in the ACR-156 phantom displayed on respective LCDs. As shown in these captured images, the noise reduction effects of the SHR-LCDs were perceptually confirmed in the agreement with the NPS results. 


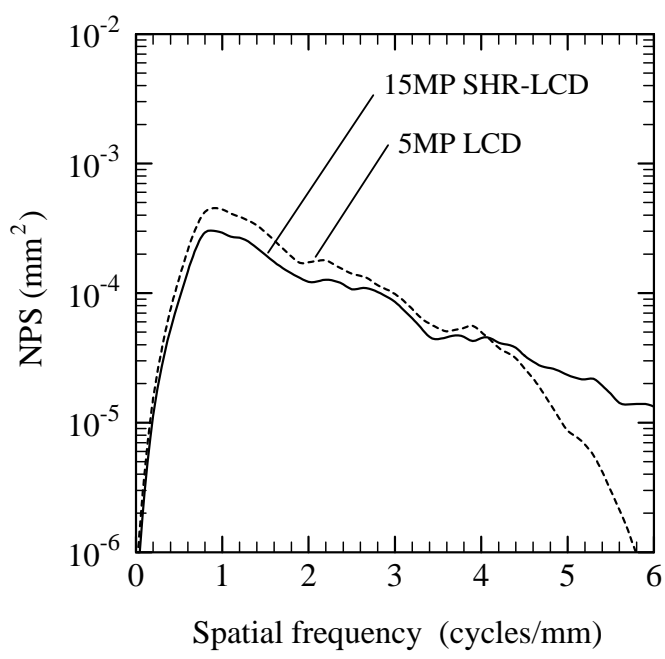

(a)

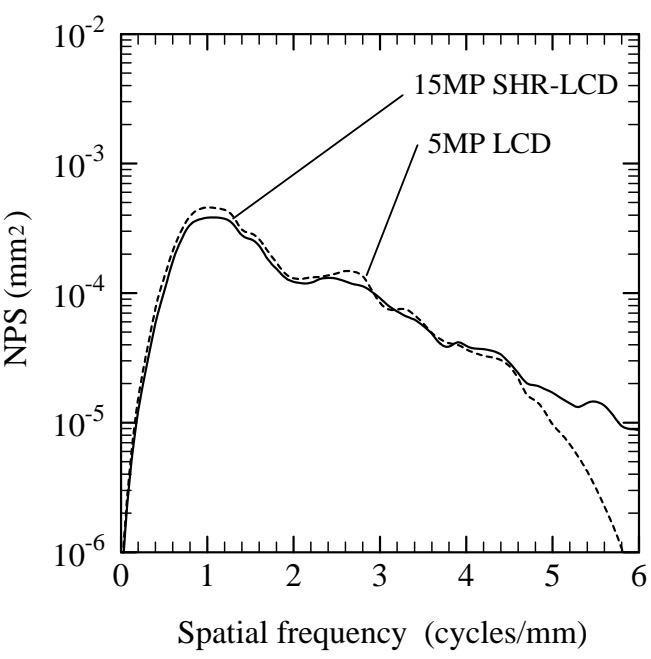

(b)

Fig.6. Noise improvement on 15MP SHR-LCD with the sub-sampling ratios of (a) 0.45 and (b) 0.55 . The phantom image obtained by Profect CS was used.

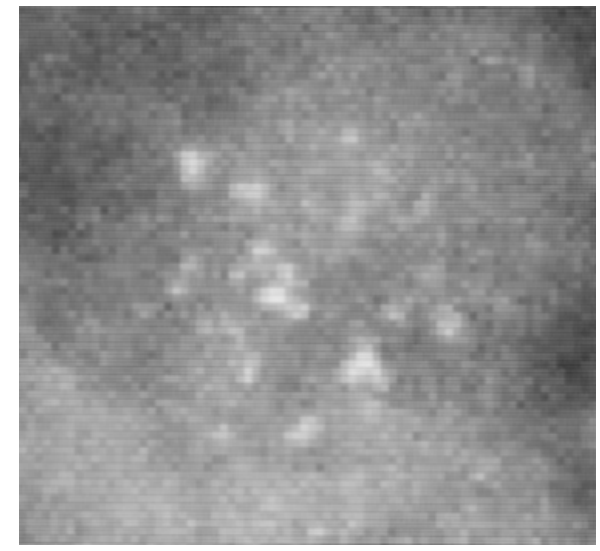

(a)

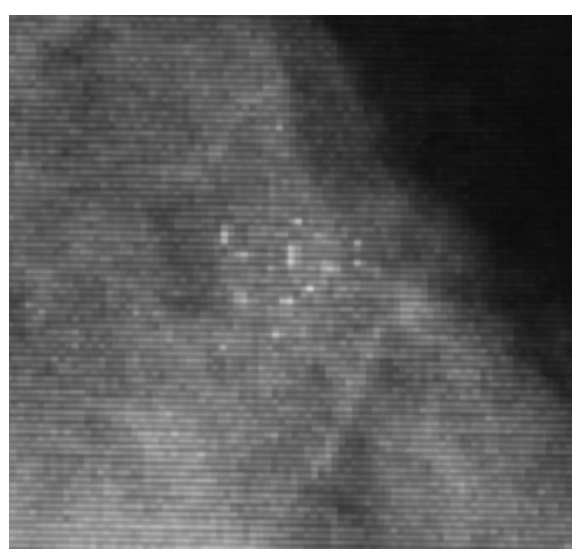

(c)

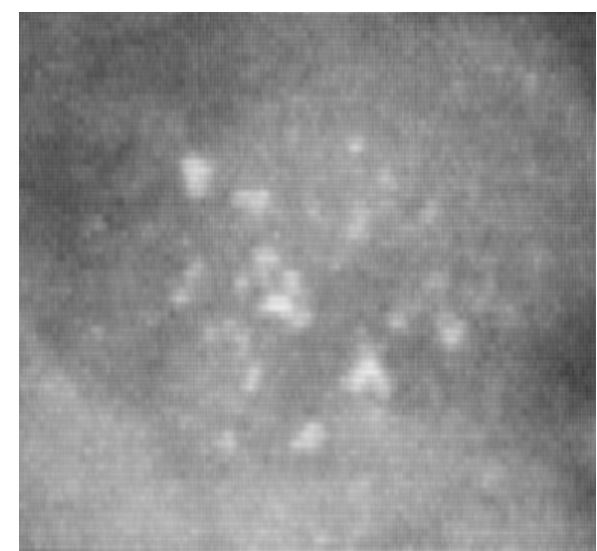

(b)

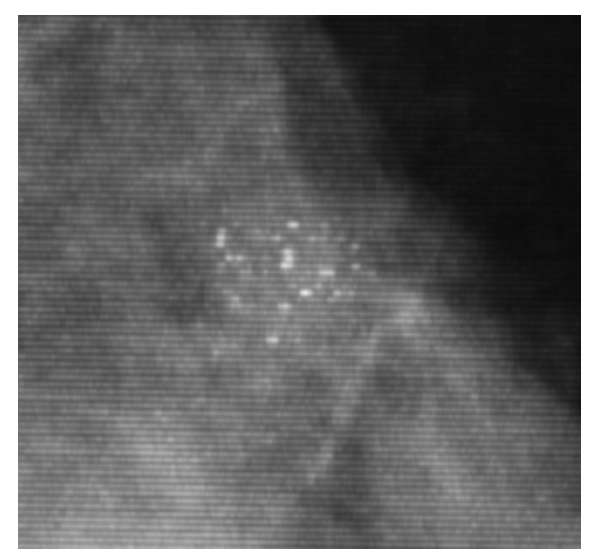

(d)

Fig.7. Comparison of the captured micro-calcification images in the clinical mammograms displayed on (a) 5MP LCD, (b)15MP SHR-LCD, (c)2MP LCD and (d) 6MP LCD. 

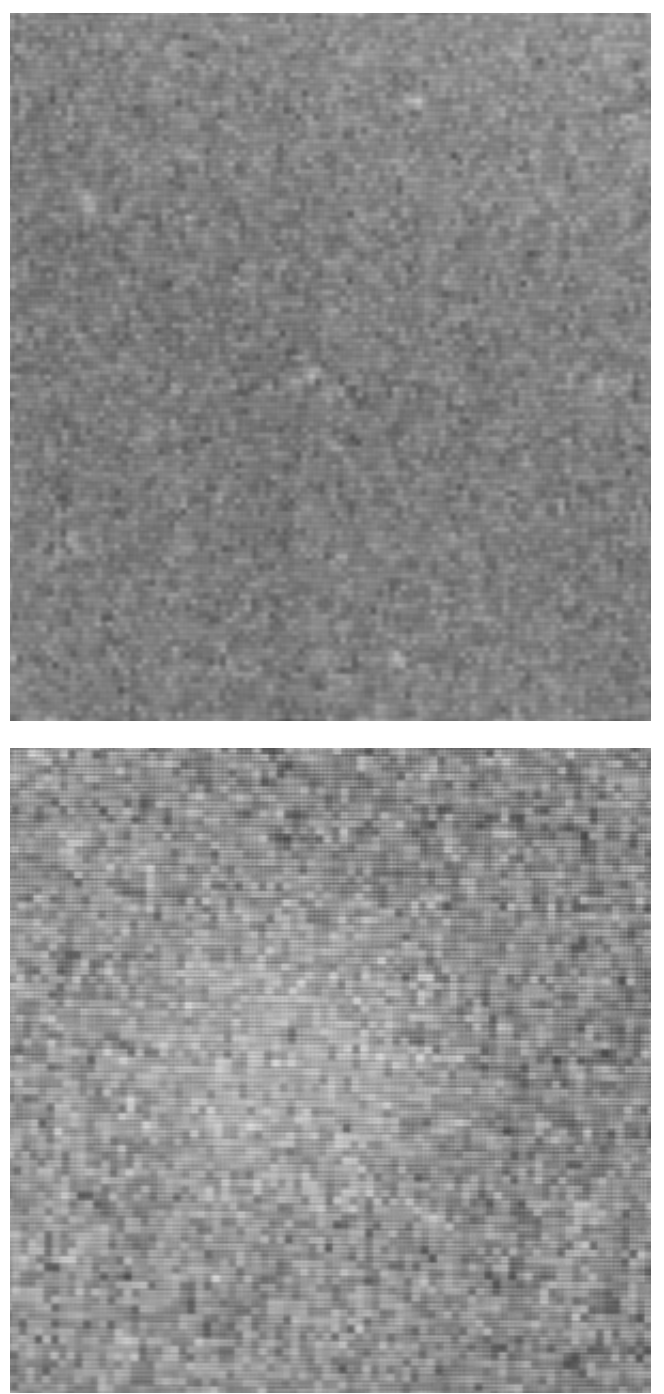

(a)
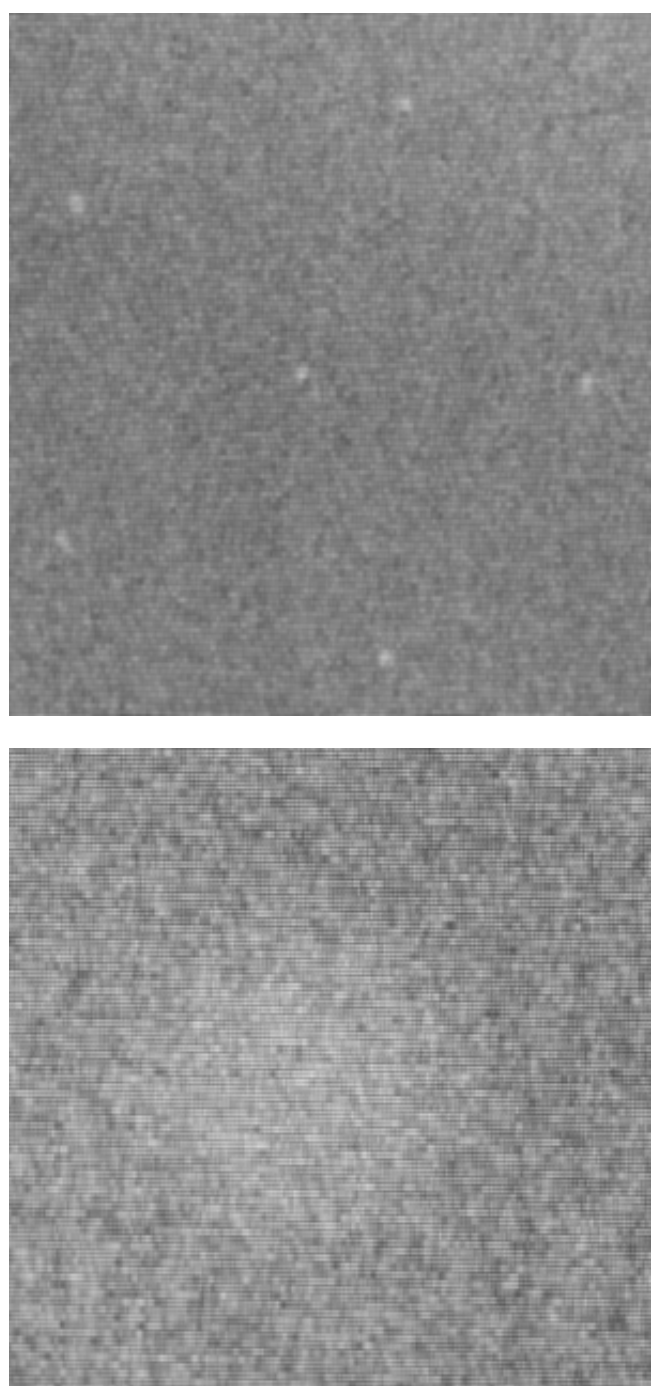

(b)

Fig.8. Captured test object's images in the ACR-156 phantom image displayed on (a) 5MP LCD and (b)15MP SHR-LCD

\section{DISCUSSION}

In displaying uniform data image such as test pattern with a specified DDL, the normal mode drives their pixels at the uniform drive levels including levels of each sub-pixel as well as those of ISD mode. Therefore, the drive levels of the sub-pixels for both modes are perfectly equivalent, and thus the ISD does not affect the native noise property of the LCD. This principle was proved by the fact that the resultant native NPSs of both modes showed the agreements.

The viewing software used in this study had a function to switch the normal mode and the ISD mode immediately. When we observed the images that contain recognizable noise by the normal mode and then we 
switched the mode to the ISD mode, we could easily find that many of the displayed image noise had been wiped off. This phenomenon supported the differences of the NPSs between the normal mode and the ISD mode.

As shown in the NPS results of Fig.4 and Fig.5, the noise levels of the ISD mode at high frequency region indicated the higher levels than those of normal mode. But the noise components with such high frequencies are almost invisible due to the frequency response of human eyes. In addition, as shown in all the NPS results, the noise levels in the high frequency region were lower than those in the low frequency region. Therefore we considered that enough noise reduction effect could be felt by our observations. In the sub-sampling ratio more than 0.55 , the noise reduction by the ISD mode did not occurred as shown in Fig.5. This result indicated that the noise reduction effects are brought by the noise-aliasing reduction, which is provided by the high frequency data sampling implemented for sub-pixel rendering.

From the perceptual comparisons by using the clinical micro-calcification images and the test object images in the ACR-156 phantom, remarkable noise reduction effects could be observed. In the normal mode, the displayed noise in each image was un-natural because the noise images were polluted by the aliasing. In contrast, the ISD mode could overcome these pollutions by the sub-pixel rendering (tripled frequency sampling). In spite of the lower noise level of the ISD mode, we could recognize the finer noise patterns represented on SHR-LCD's screens when displaying the clinical images. However the noise patterns had the lower levels and more natural appearances because of less noise aliasing. Therefore, such noise patterns did not have nagative influence on the image quality, and the resolution improvement by the ISD became more effective.

\section{CONCLUSION}

We proved the noise reduction effect of the SHR-LCD by means of the noise power spectrum measurements and the perceptual comparison of phantom and clinical images, and revealed that the ISD technology improved not only the resolution property but also the noise property of the displayed image on the LCD. We believe that the SHR-LCDs equipped with the ISD technology have the excellent ability to display the high-resolution clinical images.

\section{REFERENCES}

[1] Ichikawa K, Kodera Y, Nishi Y, Hayashi S, Hasegawa M: Development of a new resolution enhancement technology for medical liquid crystal displays, Proc. SPIE, 6516, 65160W1-65160W 8, 2007

[2] Ichikawa K, Horii A, Kodera Y: Novel NPS measurement method for medical liquid crystal display using periodic components subtraction technique, Proc. SPIE, 6142, 61423H1-61423H8, 2006

[3] Giger ML, Doi K, and Metz CE: Investigation of basic imaging properties in digital radiography. 2. Noise Wiener spectrum, Med Phys, 11(6), 797-805, 1984.

[4] Giger ML, Doi K, and Fujita H: Investigation of basic imaging properties in digital radiography. 7. Noise Wiener spectra of II-TV digital imaging systems, Med Phys, 13 (2) , 131-138, 1986.

[5] Siewerdsen JH, Antonuk LE, El-Mohri Y, et al.: Signal, noise power spectrum, and detective quantum efficiency of indirect-detection flat-panel imagers for diagnostic radiology, Med Phys, 25(5), 614-628, 1998.

[6] Ichikawa K, Kodera Y, Fujita H: MTF measurement method for medical displays by using a bar-pattern Image, Journal of SID, 14(10), 831-837, 2006 